\title{
Research on the Fault Diagnosis of Power Battery Based on Comprehensive Fuzzy Evaluation
}

\author{
Haiying Wang ${ }^{1}$, Peng $\mathrm{Liu}^{1}$, Yu Zhao ${ }^{1}$, Lei $\mathrm{Du}^{1}$ and Gechen $\mathrm{Li}^{2}$ \\ ${ }^{1}$ School of Automation, Harbin University of Science and Technology, \\ Harbin, China \\ $R \& D, C E N S$ Energy-Tech Co. Ltd., Hangzhou, China \\ wanghy@hrbust.edu.cn
}

\begin{abstract}
In the actual application process, power battery may not behave properly, such as insufficient charging, internal resistance increment, capacity reduction and etc, the paper establishes a fuzzy comprehensive evaluation model of power battery to diagnose judge and analyze. The diagnostic results are given in the form of battery health status DOH and maintenance information. The paper tests the model through Actual working conditions of electric vehicle, and the results show that the actual fault information is consistent with the diagnostic results of model. The model is reasonable, and it can be used to diagnose the faults of power battery.
\end{abstract}

Keywords: power battery; comprehensive fuzzy evaluation; health status

\section{Introduction}

Recently, more and more power battery systems are being widely used in various industry applications, due to consideration of energy development energy safety and environment Protection [1]. The normal operation of power battery directly affects the safe and reliable operation of equipment. Therefore, to diagnose the faults of battery timely and accurately is particularly important $[2,3]$. Against this background, Fault diagnosis technology is developed rapidly [4].

At present, the fault diagnosis methods which are widely applied are as follow: the diagnosis method based on wavelet transform, based on the estimated fault signal modal, based on expert systems, knowledge engineering, fuzzy technology, neural network technology and other aspects of the fault diagnosis method [5]. Foreign develops rapidly in the fault diagnosis of power battery .They have researched some fault diagnosis systems, such as BADICHEQ, BADICOCH, BAITMAN, these systems can test the performance of battery, then giving the diagnostic results which battery can be judged good or bad, and to find the error condition of battery [6, 7], our countries fault diagnosis technology which is relatively mature, such as intelligent expert diagnosis technology, the diagnostic techniques of vehicle[8], but these diagnostic techniques have their own limitations in the fault diagnosis of battery.

In this paper, we take fuzzy comprehensive evaluation method to diagnose the faults of the battery. With the battery-related data, the membership degrees value of the symptoms can be calculated, and then find the membership degree value of the faults by the fuzzy relation matrix, using the value reflects the tendency of various faults, and finally judge the faults of battery.

\section{The Fuzzy Comprehensive Evaluation Model of Power Battery}

Currently, the description of the membership degrees value of the symptoms and thinking of expert diagnosis is uncertain and vague, if we want to accurately 
describe the relationship between symptoms and faults, it is unrealistic. By fuzzy logic, using inexact reasoning method to establish fault diagnosis model, it can make the fault diagnosis module of power battery simulating expert reasoning thinking better, so as to diagnose the faults of battery accurately.

In this paper, it uses fuzzy comprehensive evaluation model to diagnose the faults of battery. The follow, the theoretical basis of this method is introduced. The battery symptoms is kinds and faults causes is kinds, using vector expresses them.

The fuzzy vector of symptoms is:

$$
X=\left(x_{1}, x_{2}, \ldots, x_{m}\right)
$$

The fuzzy vector of faults causes is:

$$
Y=\left(y_{1}, y_{2}, \ldots, y_{n}\right)
$$

The relationship between $\mathrm{Y}$ and $\mathrm{X}$ is:

$$
\boldsymbol{Y}=\mathbf{X} \boldsymbol{R}
$$

This equation can reflect the fuzzy relationship between fault causes and symptoms. The vector of symptoms can be obtained by calculating the function of membership. "o" is fuzzy operator, $\mathrm{R}$ is fuzzy relation matrix. Its value can be obtained by the experience of battery experts and knowledge.

$$
R=\left[\begin{array}{cccc}
r_{11} & r_{12} & \cdots & r_{1 n} \\
r_{21} & r_{22} & \cdots & r_{2 n} \\
\cdots & \cdots & \ddots & \vdots \\
r_{m 1} & r_{m 2} & \cdots & r_{m n}
\end{array}\right]=\left(r_{i j}\right)_{m \times n}
$$

Among them, $0 \leq r_{i j} \leq 1,1 \leq i \leq m, 1 \leq j \leq n$.

The relationship between the fault symptoms and causes can be described by the fuzzy relation matrix $\mathrm{R}$, start with equation (3).

$$
\begin{aligned}
& Y=X \circ R
\end{aligned}
$$

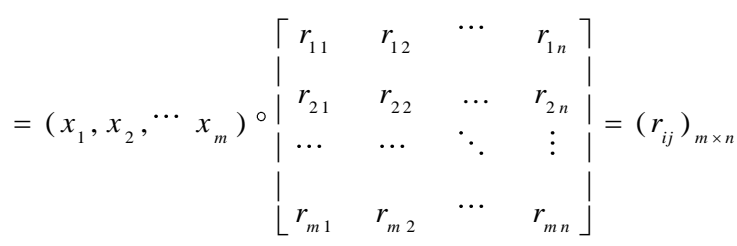

In order to solve $\mathrm{Y}$, the fuzzy operator “o "is rewritten into the following form:

$$
y_{i}=\mathbf{U}_{i=1}^{m} x_{i} r_{i j}, j=1,2, \mathrm{~L}, n
$$

When $x_{i}(i=1,2, \cdots, m)$ is normalized, at the same time, $\sum_{i=1}^{m} x_{i}=1$, it can be resulted that $\mathrm{U}_{i=1}^{m} x_{i} r_{i j}=\sum_{i=1}^{m} x_{i} r_{i j} \leq 1$ from $0 \leq r_{i j} \leq 1$, and then, fuzzy operator" o "will change into a form of real numbers together. Model will be converted into a matrix operations. As shown below: 


$$
y_{j}=\sum_{i=1}^{m} x_{i} r_{i j}, j=1,2, \mathrm{~L}, n
$$

While

$$
\sum_{i=1}^{m} x_{i}=1
$$

The fuzzy diagnosis model of battery is based on the above. Fuzzy diagnosis process is shown in Figure 1.

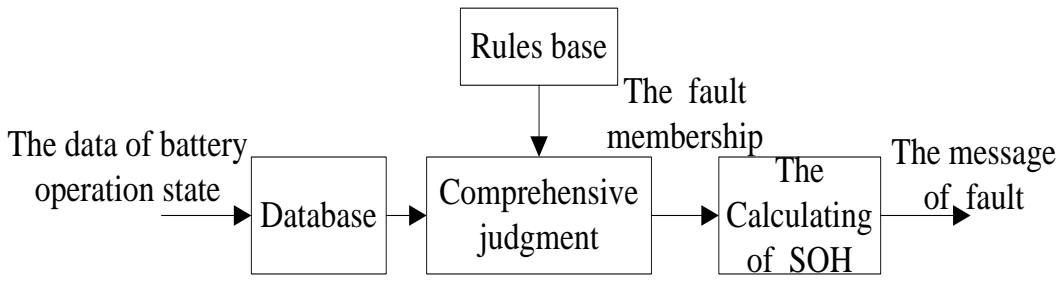

Figure 1. Fuzzy Diagnosis Progress

\subsection{The Membership Degree of Symptoms is Calculated}

Because of the complexity when the electric vehicle is running, it is difficult to use a specific mathematical model to describe the degree of change by the data of battery. This topic research through a lot of bench tests and vehicle experiments combined with battery expert experience, summed up a set of more practical calculation method, the method in the fault diagnosis is most frequently used and most representative, the specific form is as follows:

1. The first type is $\frac{B}{A}$. Symptoms is described as followed when it is charged, the voltage of cell is high. When it is discharged, the voltage of cell is low. The method is as follows.

$$
e=\frac{x_{i}-\bar{x}}{\bar{x}}
$$

where, $e$ is the average value between Individual value and the difference, $x_{i}$ is battery is a single voltage value (such as monomer voltage), $\bar{x}$ is the average over all data.

2. The second type is $\frac{\mathrm{VB}}{\mathrm{VA}}$. This kind of symptom type of battery describes the parameter rate of battery, such as: the voltage of cell grows fast when it is charged, the voltage of cell drops fast when it is discharged, the temperature of cell rises fast when it is discharged etc. This type can use the following methods:

$$
e=\frac{\mathrm{V} x_{i}-\mathrm{V} \bar{x}}{\mathrm{~V} \bar{x}}
$$

Among them, 


$$
\begin{aligned}
& \mathrm{V} x_{i}=x_{i 2}-x_{i 1} \\
& \mathrm{~V} \overline{x_{i}}=\overline{x_{2}}-\overline{x_{1}}
\end{aligned}
$$

$x_{i 1}$ and $x_{i 2}$ are the cell value of the first time and the second time after two consecutive test(such as: the voltage of cell), $\bar{x}_{1}$ and $\overline{x_{2}}$ are the average value of the first time and the second time after two consecutive test, by the above two kinds of methods, the value of $e$ can be calculated, then by introducing a reference coefficient, the membership degree of symptoms $\mu_{s}$ can be calculated, while:

$$
\mu_{s}= \begin{cases}\left|\frac{e}{C}\right| & 0 \leq\left|\frac{e}{C}\right|<1 \\ 1 & \left|\frac{e}{C}\right| \geq 1\end{cases}
$$

$\mathrm{C}$ is the reference coefficient that is, each symptom corresponds to a coefficient value, $\mu_{s}$ is a membership degree of battery symptoms.

Diagnosis model of this study is based on the membership degree of symptoms, that is, the precision of the membership degree value of symptoms will influence the calculation of the membership degree of faults. By above it can see, the reference coefficient affect the value of the membership degree of faults, and its value is obtained through a large number of experiments, based on the predecessors experience the value of $\mathrm{C}$ can be obtained.

\subsection{Calculating the Membership Degree of Faults and Fuzzy Relation Matrix}

Fuzzy relation matrix $\mathrm{R}$ is the core of fuzzy comprehensive evaluation model. The value of the elements in the matrix has a direct influence on accuracy of diagnosis. So, the value of fuzzy diagnosis matrix must be determined reasonable, scientific, and careful. Fuzzy relation matrix generally is established in two phases:

(1) To judge and set the initial value by the expert experience.

(2) To revise the $R$ values of the matrix during the diagnosis, referring the experience.

This paper obtaining the $\mathrm{R}$ values of fuzzy diagnosis matrix relies mainly on expert experience. In the following we will describe the method that how to solve the membership degree of faults. There, let a certain fault $\mathrm{F}$ relate to $\mathrm{n}$ symptoms $\mathrm{s}$, $\mu_{s_{1}}, \mu_{s_{2}}, \ldots \mu_{s_{n}}$ are the membership degree of $\mathrm{n}$ symptoms in the fuzzy relation matrix the symptoms and the faults is corresponding. $C_{1}, C_{2}, \ldots C_{n}$ are the corresponding elements. The added sum of $C_{1}, C_{2}, \ldots C_{n}$ is one. The membership degree of faults $\mu_{F}$ can be obtained by the equation 14 .

$$
\mu_{F}=C_{1} \mu_{s_{1}}+C_{2} \mu_{s_{2}}+\ldots+C_{n} \mu_{s_{n}}
$$

When the battery is used, the faults usually occurs with different symptoms, the element value of fuzzy relation matrix $R$ can reflect the size of effect that symptoms $\mathrm{n}$ to faults $\mathrm{F}$. Therefore, taking a matrix element value as the weight value of faults, equation 14 is the form of the membership degree of faults, and then it can reflect the actual fault condition of battery. 


\subsection{DOH and DOF are Determined}

The fuzzy vector $Y=\left(y_{1}, y_{2}, \ldots y_{n}\right)$ can be obtained by $Y=X \circ R$, the judgment of battery faults generally follows three kinds of principles:

(1) The method of maximum membership judgment.

(2) The method of threshold restriction.

(3) Closeness method. The method of maximum membership judgment is used in this model.

The symptoms belong to the given domain U. the fuzzy subset of $\mathrm{n}$ faults is $A_{1}, A_{2}, \ldots A_{n}$, among them $\mu_{n} \in U$, therefore the fault membership of $\mathrm{n}$ fuzzy subset is $\mu_{A_{1}}\left(\mu_{o}\right), \mu_{A_{2}}\left(\mu_{o}\right), \ldots, \mu_{A_{n}}\left(\mu_{o}\right)$, if $i \in\{1,2, \ldots, n\}$ can make the equation (15) established that.

$$
D O F=\mu_{A_{i}}\left(\mu_{o}\right)=\max \left(\mu_{A_{1}}\left(\mu_{o}\right), \mu_{A_{2}}\left(\mu_{o}\right), \ldots, \mu_{A_{n}}\left(\mu_{o}\right)\right)
$$

$\mu_{\mathrm{o}}$ is subordinated to $A_{i}$, the value of DOF is the max among the membership of fuzzy membership. Then current fault of battery can be determined.

The value of DOF is judged by the method of maximum membership judgment, in this way, the judgment is easy and programming is easy to implement, but when the value of DOF is judged, excluding the relatively small membership that bring actual impact, so this method outlines less information.

In order to classify the battery, when battery is using whose performance is good or bad, the DOH of battery is introduced. When the electric vehicle is running, it will appear with complex conditions, so the voltage and current data of battery will change rapidly, this changing will conduct to the DOF mutation. DOH can reduce the occurrence of mutation. In this model, DOH is divided into ten levels, as shown in Table 1.

Table 1. The Grade Classification of DOH

\begin{tabular}{c|c}
\hline The grade of DOH & The define of classification \\
\hline $1-3$ & Replace the battery which is in fault \\
\hline $4-6$ & Maintain the battery \\
\hline $7-10$ & The battery is healthy \\
\hline
\end{tabular}

DOH can be obtained by the equation 16 .

$$
D O H=C_{1} \mathrm{~g}(1-D O F)+C_{2} \mathrm{~g} D O H_{1}+C_{3} \mathrm{~g} D O H
$$

In the equation $16, \mathrm{DOH}_{1}$ and $\mathrm{DOH}_{2}$ are obtained from history database, they are the $\mathrm{DOH}$ of last one and last two, then $\mathrm{DOH}$ is obtained by the weighted sum of $\mathrm{DOH}_{1}, \mathrm{DOH}_{2}$ and DOF. $\mathrm{C}_{1}, \mathrm{C}_{2}$ and $\mathrm{C}_{3}$ are the weighting coefficients in the equation. The sum of $\mathrm{C}_{1}, \mathrm{C}_{2}$ and $\mathrm{C}_{3}$ is one. Based on normalization method and experiment, it set the value of $\mathrm{C}_{1}, \mathrm{C}_{2}$ and $\mathrm{C}_{3} . \mathrm{C}_{1}$ is $0.5, \mathrm{C}_{2}$ is 0.3 , then $\mathrm{C}_{3}$ is 0.2 . Finally, the current value of DOH is stored in the historical database for the next calculation.

\subsection{Building the Knowledge Base of Fault Diagnosis}

The knowledge base of fault diagnosis is composed by database and rule base. The database is used for storing the diagnostic status data of battery, the rule base is 
used for storing rules that are needed in model.

In the fault diagnosis module, database stores battery's operation status data and history data which are significant. The state data are mainly as follows: the total voltage, total current, soc, cell voltage and temperature and etc. In the model, History database is mainly used for saving the fault records when battery is running on the vehicle, it also can save the last two values of DOH. The History database records need to be continuously updated, in this paper, for the dynamic performance data of battery, it take the way to update regularly.

\section{The Design of Fault Diagnosis Reasoning Module}

The function of reasoning module can deduce the final result. It makes use of the symptoms data which is based on the user, selecting the relevant knowledge from the knowledge base, according to a certain logic reasoning strategy to deduce, finally, it achieves the final result.

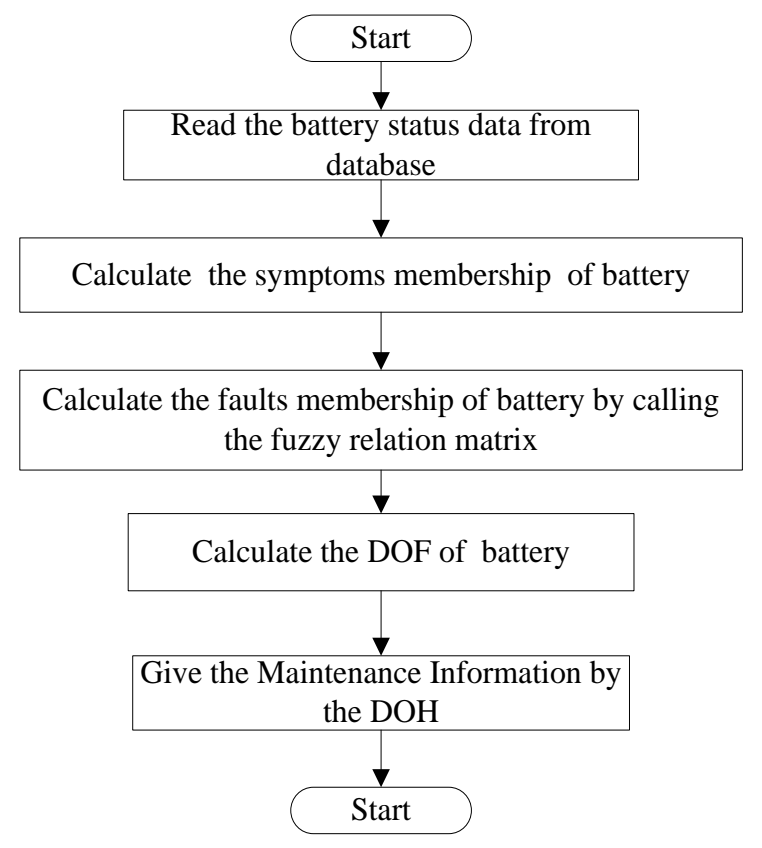

Figure 2. The Flow Chart of Reasoning Module

This model uses a forward imprecise reasoning method. At first, the reasoning module reads the status data of battery from the database. then, using the function formula of rules base calculates the symptom membership of battery $\mu_{s}$, by the rule which is $Y=X \circ R$, the membership degree of faults can be calculated and obtained, finally, by calculating the DOF and the DOH of battery, the fault of battery can be showed. The overall chart of reasoning module is showed in Figure 2.

\subsection{The Instructions of System Verification}

After building the fuzzy comprehensive evaluation model, this paper conducts system verification in order to verify the accuracy of model. The system verification contains two parts, one is bench test, and the other is vehicle test. Before testing, using two cells whose fault type has been determined replaces two healthy cells. The others are healthy in order to keep that the overall performance of battery is good. The specific method is showed in Table 2. 
Table 2. The Test Description

\begin{tabular}{c|c|c}
\hline The type of test & Bench test & Vehicle test \\
\hline The type of battery & $\begin{array}{c}\text { Five single lithium iron phosphate } \\
\text { battery }\end{array}$ & $\begin{array}{c}20 \text { groups lithium iron } \\
\text { phosphate battery pack }\end{array}$ \\
\hline equipment & $\begin{array}{c}\text { The ZEEMOO8960 test system } \\
\text { which is produced by ZM company }\end{array}$ & $\begin{array}{c}\text { hybrid electric bus } \\
\text { which is produced by } \\
\text { CENS Energy-Tech } \\
\text { Company }\end{array}$ \\
\hline The test method & $\begin{array}{c}\text { Under normal temperature,the } \\
\text { battery is discharged in the constant } \\
\text { current which is 0.3C、 0.5C、 } \\
\text { 1C,after laying aside some time, all } \\
\text { the battery is charged in the constant } \\
\text { current which is 0.5C.At the end of } \\
\text { charging, the fault diagnosis } \\
\text { message is obtained, the message is } \\
\text { the final result. }\end{array}$ & $\begin{array}{c}\text { At first the battery is } \\
\text { charged, then the } \\
\text { Vehicle test is } \\
\text { conducted, until all the } \\
\text { power is depleted, the } \\
\text { fault diagnosis message } \\
\text { is obtained, the message } \\
\text { is the final result. }\end{array}$ \\
\hline
\end{tabular}

The program of test is as follow: at first, using two fault batteries which are marked with NO.1 and NO.2 battery replace the NO.2 and NO.4 battery among the five healthy battery. Then by the bench test, the battery fault can be obtained. Comparing the diagnostic result of model with the result of bench test, it can verify if the result is consisted or not.

Generally, battery pack consist of eight layer single battery which are in series, the performance of battery is good or bad depending on the performance of the worst single battery. In the vehicle test, the two fault battery whose fault type has been ensured are put into two battery pack, the other battery pack are healthy. Then conducting the vehicle test, the diagnostic fault of model will be verified.

\subsection{The Curve of Test and the Analysis of Diagnostic Result}

The general fault analysis for No.1 battery can be obtained that battery capacity reduction, charging insufficiency and over-increment in internal resistance may occur resulting in faults above. Apart from the facts that the charging voltage may be lower than the average value when battery charging is insufficient and that the voltage may exceed the average value during charging section and lower than the average value in discharging section when the internal resistance is over-increased. The general fault for No.1 battery can be focused on capacity reduction from the symptom described in the figures with rapid voltage reduction in discharging section and rapid voltage increment in charging section.

Figure 3 and 4 has described the charging and discharging curve under different rates respectively. It can be deduced from the figures that the voltage variation rate of fault batteries has fairly kept consistent with that of normal batteries and the voltage reduction of No.1 battery has exceeded that of average voltage under different discharging rates in general with lower voltage as well. In addition, the variation rate of voltage of the fault battery has also exceeded that of the average voltage with higher voltage.

During the process of charging and discharging, the total voltage of NO.2 single battery is lower than the average voltage. From the figure of $0.5 \mathrm{C}$ rate constant current discharge, it can be seen that the voltage rate drop significantly faster than the average at this stage, after analyzing, the cause of fault may be due to battery capacity. 


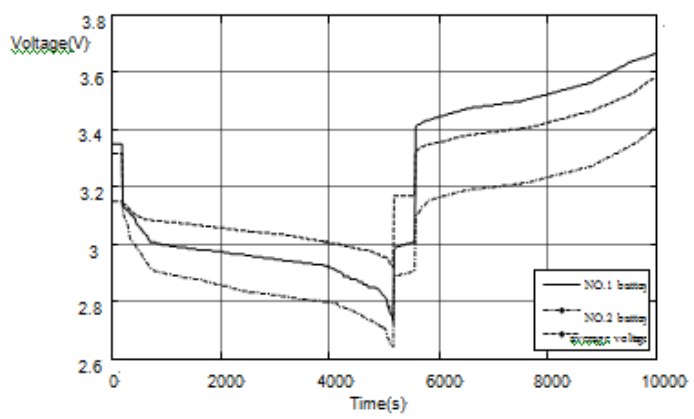

Figure 3. The Curve of $0.5 \mathrm{C}$ Constant Current Discharging and $0.5 \mathrm{C}$ Constant Current Charging

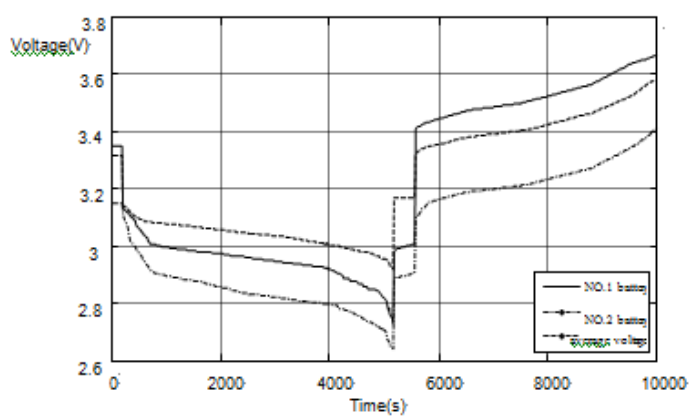

Figure 4. The Curve of $1 \mathrm{C}$ Constant Current Discharging and $0.5 \mathrm{C}$ Constant Current Charging

At the stage of $0.5 \mathrm{C}$ rate constant current charging, the voltage curve of NO.2 single battery shows that its monomer value is low and rises faster. By experience, if the curve shows that the charging voltage is high, it may be caused by capacity small or internal resistance larger. If the self-discharge rate of battery is high, it usually leads to that the charging voltage changes slowly and the static voltage drop faster .Therefore, by the empirical analysis, it determines that the fault of NO.2 battery is insufficient charging.

After all test finishing, the diagnostic results are given by the program of fault diagnosis. It can be seen in the Table 3. The result of battery diagnosis is consistent with the real situation.

Table 3. The Diagnosis Results of Bench Test

\begin{tabular}{c|c|c}
\hline Type of fault & $\begin{array}{c}2 \\
\text { battery one }\end{array}$ & $\begin{array}{c}4 \\
\text { battery two }\end{array}$ \\
\hline capacity reduction & 0.78 & 0.51 \\
\hline battery damage & 0.13 & 0.17 \\
\hline insufficient charging & 0.34 & 0.86 \\
\hline the self-discharging increment & 0.23 & 0.36 \\
\hline internal resistance increment & 0.61 & 0.41 \\
\hline The grade of DOH & 3 & 2 \\
\hline
\end{tabular}


Before the test of vehicle, using two fault battery pack which are marked replace the NO.7 and NO.13 healthy battery pack, the other battery pack are healthy. The road state is complex when Electric bus is running, so it will make the voltage and current change instantly. So the DOH grade of vehicle test is lower than the bench test. Overall, the diagnostic results of vehicle test are consistent with the actual judgment. Table 4 shows the comparison of fault membership between the fault battery and the healthy battery.

Table 4. The Fault Diagnosis Results of Vehicle Test

\begin{tabular}{c|c|c|c|c|c}
\hline Code & 3 & 7 & 11 & 13 & 20 \\
\hline Type of fault & & & & & \\
\hline capacity reduction & 0.03 & 0.91 & 0.13 & 0.67 & 0.05 \\
\hline battery damage & 0.01 & 0.16 & 0.06 & 0.15 & 0.03 \\
\hline insufficient charging & 0.06 & 0.44 & 0.16 & 0.93 & 0.14 \\
\hline the self-discharging increment & 0.02 & 0.16 & 0.07 & 0.05 & 0.02 \\
\hline internal resistance increment & 0.07 & 0.69 & 0.05 & 0.52 & 0.05 \\
\hline The grade of DOH & 10 & 2 & 9 & 1 & 9 \\
\hline
\end{tabular}

\section{Concluding Remarks}

For the fault diagnosis of power battery is complex, this paper establishes the fuzzy comprehensive evaluation model of fault basing on the fuzzy reasoning method. By determining the function of symptom membership, the diagnostic rules of battery and the fuzzy relation matrix, the fault membership can be calculated and obtained. The fault membership is the basis of battery fault diagnosis. The realization of fault diagnostic module refers to the expert system's development process. The diagnostic model of battery is verified by the data which is in the actual condition. The model can diagnose the fault of battery truly, the model can be applied in the reality.

\section{Acknowledgments}

This paper is partially supported by Technological Innovation Foundation for Leaders of Disciplines in Science of Harbin (2014RFXXJ032).

\section{References}

[1] H. Wang, L. He, X. Qi, H. Wang, G. Li and X. Dai, "Simulation system of the performance of power battery for electrical vehicle based on internet of things", 2012 International Conference on Measurement, Information and Control(MIC 2012), vol. 2, (2012), pp. 681-684.

[2] X. Yun, Z. Zhong, Z. Sun, T. Zhang and T. Yin, "Research on fault diagnosis method of FCV power battery based on physical model", 2011 1st International Conference on Mechanical Engineering(ICME 2011), vol. 52-54, (2011), pp.1438-1444.

[3] K. Kim, S. Hwang, K. Sung, and Y. Kim, "A study on the fault diagnosis analysis of variable reluctance resolver for electric vehicle", IEEE Sensors 2010 Conference, (2010), pp. 290-295.

[4] C. Song, J. Li, D. Qu, D. Zhou and L. Fan, "Online fault diagnosis of hybrid electric vehicles based on embedded system", 2010 International Conference on Computer, Mechatronics, Control and Electronic Engineering(CMCE 2010), vol.4, (2010), pp. 274-277.

[5] A. Hiba and M. Nazih, "Fault detection and diagnosis of renewable energy systems: An overview", 2012 International Conference on Renewable Energies for Developing Countries( REDEC 2012), (2012).

[6] H. Mohammed, "Vehicle valve regulated lead acid battery modeling and fault diagnosis", SAE 2010 World Congress and Exhibition, (2010).

[7] H. Wang, H. Fu, Y. Liu, Y. He and G. Li, "Research in the equilibrium control of power battery pack of Li-ion”, ICIC Express Letters, Part B: Applications, vol.4, no.3, (2013) June, pp. 617-624.

[8] S. Souahlia, K. Bacha, A. Chaari, Power transformer fault diagnosis based on dissolved gas analysis by artificial neural network, 2012 1st International Conference on Renewable Energies and Vehicular Technology,(REVET 2012), (2012), pp. 230-236. 
International Journal of Control and Automation Vol. 9, No. 4 (2016) 